\title{
Estudio analítico y experimental del desempeño de motores diésel alimentados con bio-oil hidrotratado
}

\section{Analytical and experimental performance study of diesel engines powered with hydrotreated Bio-Oil}

\author{
Gabriel Fernando García-Sánchez ${ }^{1}$, Jorge Luis Chacón-Velasco², Arlex Chaves-Guerrero ${ }^{3}$, Andrés Felipe \\ Lopez-García ${ }^{4}$
${ }^{1}$ Grupo de Investigación en Desarrollo Tecnológico, Mecatrónica y Agroindustria (Gidetechma), Facultad de Ingeniería Mecánica, Universidad Pontificia Bolivariana, Colombia. Email: gabrielf.garcia@upb.edu.co Santander, Colombia. Email: jchacon@uis.edu.co Santander, Colombia. Email: achavesg@uis.edu.co
${ }^{4}$ Grupo de Manejo Eficiente de la Energía (Gimel), Facultad de Ingeniería, Universidad de Antioquia, Colombia. \\ ${ }^{2}$ Grupo de Investigación en Energía y Medio Ambiente (Giema), Escuela de Ingeniería Mecánica, Universidad Industrial de \\ ${ }^{3}$ Grupo de Investigación en Energía y Medio Ambiente (Giema), Escuela de Ingeniería Química, Universidad Industrial de \\ Email: anfelo77@gmail.com
}

Recibido: octubre 20, 2017. Aceptado: febrero 27, 2018. Versión final: marzo 30, 2018.

\begin{abstract}
Resumen
Debido a los graves problemas presentados a causa del amplio uso de biocombustibles de primera generación, los biocombustibles de segunda generación se presentan como una posible mejor alternativa para la sustitución de los combustibles convencionales provenientes del petróleo. En el presente artículo se presenta un estudio del desempeño de motores diésel alimentados con bio-oil hidrotratado proveniente de biomasa lignocelulósica, el cual es un biocombustible de segunda generación; para esto se desarrolló un modelo termodinámico cero-dimensional de la combustión que predice los parámetros de desempeño del motor. Este modelo fue validado contra datos experimentales de presión en cámara de dos motores diésel alimentados con mezclas diésel/biodiésel y diésel/bio-oil hidrotratado, observándose una buena correlación entre los datos simulados y experimentales, con errores relativos promedio en los valores pico de presión de 0,5 y $1 \%$ respectivamente. Los resultados muestran que el bio-oil hidrotratado, al utilizarse en pequeñas proporciones, puede convertirse en un sustituto parcial del combustible diésel convencional.
\end{abstract}

Palabras clave: biocombustibles de segunda generación; biocombustibles de primera generación; modelos termodinámicos; simulación de motores; motores diésel.

\begin{abstract}
Due to the serious problems encountered because of the extensive use of first-generation biofuels, second generation biofuels are presented as one of the best possible alternatives conventional fuels from oil. This paper presents a theoretical study of the performance of diesel engines fueled with hydrotreated bio-oil from lignocellulosic biomass, which is a second generation biofuel. For this, a thermodynamic zero-dimensional combustion model simulation was carried out, which predicts the performance parameters of the engine. This model was validated against experimental data of two diesel engines fueled with diesel-biodiesel and diesel/hydrotreated bio-oil blends, and a good correlation
\end{abstract}

ISSN impreso: 1657 - 4583, ISSN en línea: 2145 - 8456

Este artículo puede compartirse bajo la licencia CC BY-ND 4.0 y se referencia usando el siguiente formato: G. García, J. Chacón, A. Chaves, A. López, "Estudio analítico y experimental del desempeño de motores diésel alimentados con bio-oil hidrotratado," Rev. UIS Ing., vol. 17, no. 2, pp. 115-126, 2018. Doi: https://doi.org/10.18273/revuin.v17n2-2018011 
between simulated and experimental data was observed, with average relative errors in pressure peak values of 0.5 and $1 \%$, respectively. The results show that hydrotreated bio-oil in small proportions could become a partial substitute for conventional diesel fuel.

Keywords: second generation biofuels; first generation biofuels; engine simulation; thermodynamic models; diesel engines.

\section{Nomenclatura}

$\gamma \quad$ Relación de calores específicos de la mezcla

$V \quad$ Volumen instantáneo del cilindro $\left(\mathrm{m}^{3}\right)$

$\theta \quad$ Ángulo de giro del cigüeñal ( $\left.{ }^{\circ}\right)$

$\frac{d Q_{R}}{d \theta} \quad$ Tasa de calor liberado en la combustión $\left(\mathrm{kJ} /{ }^{\circ}\right)$

$\frac{d Q_{w}}{d \theta} \quad$ Tasa de transferencia de calor a las paredes del cilindro $\left(\mathrm{kJ} /{ }^{\circ}\right)$

$\theta_{p} \quad$ Ángulo de duración de la combustión premezclada $\left({ }^{\circ}\right)$

$\theta_{d} \quad$ Ángulo de duración de la combustión por difusión

$\left({ }^{\circ}\right)$

$\theta_{o} \quad$ Ángulo de inicio de la combustión ( $\left.{ }^{\circ}\right)$

$M_{p} \quad$ Factor de forma de la ecuación de Wiebe en la combustión premezclada

$M_{d} \quad$ Factor de forma de la ecuación de Wiebe en la combustión por difusión

$Q_{p} \quad$ Calor liberado en la combustión premezclada (kJ)

$Q_{d} \quad$ Calor liberado en la combustión por difusión (kJ)

$C_{m} \quad$ Velocidad media del pistón $(\mathrm{m} / \mathrm{s})$

$R_{u} \quad$ Constante universal de los gases $(\mathrm{J} /(\mathrm{mol} \mathrm{K}))$

$n_{m} \quad$ Régimen de giro del motor $\left(\mathrm{min}^{-1}\right)$

$E A \quad$ Energía de activación aparente

$C N$ Índice de cetano del combustible

$\frac{d Q_{w}}{d t} \quad$ Tasa de transferencia de calor a las paredes del cilindro (W)

$h_{g} \quad$ Coeficiente de transferencia de calor $\left(\mathrm{W} /\left(\mathrm{m}^{2} \mathrm{~K}\right)\right)$

$T \quad$ Temperatura media del gas $(\mathrm{K})$

$T_{W} \quad$ Temperatura media de las paredes (K)

A Área de transferencia de calor $\left(\mathrm{m}^{2}\right)$

$D$ Diámetro del cilindro (m)

$P$ Presión del gas $(\mathrm{kPa})$

$V_{d} \quad$ Volumen desplazado en la carrera del pistón $\left(\mathrm{m}^{3}\right)$

$P_{m}$ Presión del motor en arrastre $(\mathrm{kPa})$

$T_{\text {ref }}$ Temperatura en el punto de referencia $(\mathrm{K})$

$P_{\text {ref }}$ Presión en el punto de referencia $(\mathrm{kPa})$

$V_{\text {ref }}$ Volumen en el punto de referencia $\left(\mathrm{m}^{3}\right)$

$C_{1}, C_{2}$ Coeficientes de la ecuación de Woschni

$T_{\text {rf }}$ Temperatura del refrigerante $\left({ }^{\circ} \mathrm{C}\right)$

$\rho_{c} \quad$ Densidad de la carga en el colector de admisión $\left(\mathrm{kg} / \mathrm{m}^{3}\right)$

pme Presión media efectiva (bar)

$L$ Longitud de la biela (m)

$S$ Carrera del pistón (m) $r$ Relación de compresión

$m f b(\theta)$ Fracción de combustible quemado

$f$ Fracción de gas residual en el cilindro

$C_{p}$ Calor específico a presión constante $(\mathrm{kJ} / \mathrm{kg} . \mathrm{K})$

$C_{v}$ Calor específico a volumen constante $(\mathrm{kJ} / \mathrm{kg} . \mathrm{K})$

$R$ Constante del gas (kJ/kg.K)

$W_{c, i g}$ Trabajo bruto indicado $(\mathrm{kJ})$

$P_{i}$ Potencia indicada $(\mathrm{kW})$

$n_{R} \quad$ Número de giros del cigüeñal en un ciclo

$\eta_{\text {ter }}$ Eficiencia térmica Indicada

$m_{f}$ Masa de combustible inyecta por ciclo $(\mathrm{kg})$

PCI Poder calorífico inferior del combustible $(\mathrm{kJ} / \mathrm{kg})$

\section{Introducción}

Debido al creciente incremento del calentamiento global y al agotamiento de las reservas de crudo ligero en el mundo, los biocombustibles de primera generación se han venido utilizando como una alternativa energética a los combustibles convencionales provenientes del petróleo. El uso de estos biocombustibles ha generado serios problemas medioambientales, como la deforestación y la escasez de alimentos [1,2], lo cual ha hecho que se despierte el interés en los biocombustibles de segunda generación, los cuales provienen de materias primas que no son fuentes alimenticias y presentan ventajas medioambientales importantes [3]. El bio-oil proveniente de residuos lignocelulósicos (pajas, residuos agrícolas e industriales, bagazo de caña, etc.) es un potencial biocombustible de segunda generación que se podría utilizar en la industria del transporte. Sin embargo. este tiene propiedades indeseables para su uso directo como combustible, como alta viscosidad, bajo poder calorífico e inestabilidad térmica [4]; por esta razón es conveniente realizar un proceso de hidrotratamiento para mejorar sus propiedades y estudiar el desempeño del producto final en aplicaciones reales.

Para evaluar el desempeño de un motor diésel alimentado por nuevos combustibles se pueden seguir dos caminos: utilizar modelos teóricos o realizar análisis experimentales. Este último es un camino que requiere de gran tiempo y esfuerzos económicos [5-7], lo cual hace que en varias ocasiones los modelos teóricos se presenten como una mejor opción. De acuerdo con Heywood [8], los modelos teóricos de la combustión se pueden 
clasificar en dos grandes grupos, modelos termodinámicos y modelos multidimensionales; los primeros buscan dar una descripción global del proceso, mientras que los segundos buscan describir en detalle cada uno de los subprocesos involucrados en el proceso de combustión diésel. Una revisión de los modelos de simulación de la combustión desarrollados alrededor del mundo se presenta en [9]. Como ejemplos de modelos multidimensionales empleados con éxito en la simulación de la combustión en motores diésel, se pueden citar los trabajos de las referencias [10-13]. Por otro lado, algunos de los trabajos desarrollados en los últimos años a partir de modelos termodinámicos han sido los de Gogoi y Baruah [6], en 2010, quienes desarrollaron un modelo con el cual se estudió el efecto de la velocidad del motor y la relación de compresión en la potencia y la eficiencia al freno de un motor diésel monocilíndrico de 4 tiempos, alimentado con mezclas de diésel y biodiésel provenientes de aceite de Karanja, Bueno et al. [14], que en el mismo año estudiaron el impacto que ocasiona el uso de éster etílico de aceite de soya en un motor diésel turbocargado por medio de un análisis energético y exergético del mismo, y Colaço et al. [15], quienes simularon el perfil de temperatura en un pistón de un motor diésel bajo varias condiciones de carga, para lo cual elaboraron un modelo termodinámico para la determinación de las respectivas condiciones de frontera. En 2012, Patil y Akarte [16] estudiaron el desempeño de un motor diésel alimentado con mezclas diésel-metil éster de aceite de palma [B0, B20, B60 y B100), analizando el efecto de la variación de la relación de compresión en la presión pico, la tasa de calor liberado y la eficiencia térmica al freno. En 2014, Kökkülünk et al. [17] utilizaron un modelo cero-dimensional de una zona para estudiar los efectos de los parámetros de diseño de un motor diésel de inyección directa, con inyección de vapor y recirculación de gases de salida (EGR), en su desempeño y emisiones de NO. En 2015, Potdukhe y Deshmukh [18] realizaron un modelo termodinámico cero-dimensional de una zona, para determinar la presión en la cámara, calor liberado y calor perdido vs. el ángulo de giro del cigüeñal y así estudiar el desempeño de las mezclas diésel-biodiésel de semilla de algodón, y Naitam y Deshmukh [19] desarrollaron un modelo cerodimensional de una zona para estudiar el desempeño de las mezclas diésel-aceite crudo derivado de semillas de Karanja. En 2016, Mikulski y Wierzbicki [20] utilizaron un modelo matemático cero-dimensional multifase de un motor de dos combustibles (gas natural/diésel) y encendido por compresión, para simular el impacto de añadir otros gases al gas natural en el proceso de combustión. Como se puede observar, en los últimos años se ha evaluado el uso de diferentes tipos de biocombustibles en motores diésel a partir de modelos termodinámicos cero-dimensionales, esto debido a que este tipo de modelo es mucho más simple que los modelos multidimensionales y tiene la capacidad de predecir adecuadamente el desempeño del motor con una alta eficiencia computacional [16].

Con el fin de conocer la afectación que tendrá el uso de bio-oil hidrotratado proveniente de residuos lignocelulósicos en el desempeño de un motor diésel, en este trabajo se desarrolló un modelo de simulación del ciclo termodinámico del motor, el cual permite conocer los principales parámetros de desempeño del motor, de una manera económica y práctica. Este modelo es adecuado para predecir el desempeño de diferentes tipos de motores diésel alimentados con cualquier tipo de combustible hidrocarburo $(\mathrm{CmHnOp})$, siempre y cuando se conozcan las características del motor, las propiedades del combustible y las condiciones de operación.

\section{Metodología}

\subsection{Descripción del modelo}

El modelo aquí presentado está basado en un balance energético de la cámara de combustión del motor. A continuación se presentan las suposiciones y ecuaciones utilizadas en su desarrollo.

\subsubsection{Suposiciones del modelo}

2.1.1.1 Presión uniforme en la cámara de combustión Esta suposición se fundamenta en el hecho de que la velocidad del fluido y la de propagación de la llama son mucho menores a la velocidad del sonido [21].

\subsubsection{El fluido en la cámara está constituido por una mezcla de aire, combustible gaseoso y productos resultantes de una combustión estequiométrica}

El considerar una mezcla estequiométrica aire/combustible para estimar la composición de los productos quemados es válida, debido a que, a pesar de operar con dosados globales pobres, la combustión ocurre en condiciones locales cercanas a la estequiométrica; es decir, la flama está localizada en la región de relación combustible-aire estequiométrica, durante la fase de quema controlada de la mezcla [22], [23].

\subsubsection{El fluido se comporta como un gas ideal}

Ya que las condiciones termodinámicas en la cámara de combustión se alejan mucho de las condiciones de saturación, esta es una suposición que resulta adecuada [20]. 


\subsubsection{La temperatura utilizada para el cálculo de las propiedades del gas es la temperatura media del cilindro}

Esta es tal vez la más restrictiva de las suposiciones, sobre todo en lo que respecta a los productos quemados al inicio de la combustión. Sin embargo, el error disminuye a medida que la combustión avanza, ya que debido a la dilución, el enfriamiento provocado por la expansión y la transferencia de calor, la distribución de temperaturas en la cámara tiende a ser más uniforme a medida que transcurre el ciclo $[19,21]$.

\subsubsection{El cilindro se considera como un sistema cerrado durante las carreras de compresión- expansión}

Esta suposición conduce a errores máximos entre la presión calculada y la experimental, que no superan el 3 $\%$ en modelos predictivos[23], como el presente, por lo que es válida para este caso.

\subsubsection{Balance de energía}

Teniendo en cuenta que la cámara de combustión se consideró como un sistema cerrado y que se asumió que el gas se comportaba como un gas ideal, en el presente modelo se utilizó la siguiente forma de la ecuación de balance de energía:

$$
\frac{d Q_{R}}{d \theta}=\left(\frac{1}{\gamma-1}\right) V \frac{d P}{d \theta}+\left(\frac{\gamma}{\gamma-1}\right) P \frac{d V}{d \theta}+\sum_{w} \frac{d Q_{w}}{d \theta}
$$

Esta ecuación es la base del modelo desarrollado ya que permite predecir el comportamiento de la presión durante el ciclo del motor. En las siguientes secciones se explica cómo se determinaron las diferentes magnitudes que la conforman.

\subsubsection{Calor liberado en la combustión}

Para determinar la tasa calor liberado en la combustión $\left(\frac{d Q_{R}}{d \theta}\right)$ se utilizó la ecuación doble de Wiebe [26]:

$$
\begin{aligned}
\frac{d Q_{R}}{d \theta}=6.9 \frac{Q_{p}}{\theta_{p}}\left(M_{p}+1\right) & \left(\frac{\theta-\theta_{o}}{\theta_{p}}\right)^{M_{p}} \cdot \exp \left[-6.9\left(\frac{\theta-\theta_{o}}{\theta_{p}}\right)^{\left(M_{p}+1\right)}\right] \\
& +6.9 \frac{Q_{d}}{\theta_{d}}\left(M_{d}\right. \\
& +1)\left(\frac{\theta-\theta_{o}}{\theta_{d}}\right)^{M_{d}} \exp \left[-6.9\left(\frac{\theta-\theta_{o}}{\theta_{d}}\right)^{\left(M_{d}+1\right)}\right]
\end{aligned}
$$

Donde los subíndices p y d hacen referencia a las fases de combustión premezclada y combustión por difusión, respectivamente.

\subsubsection{Tiempo de retraso en la ignición}

Para determinar el ángulo de inicio de la combustión $\left(\theta_{o}\right)$ es necesario conocer el tiempo de retraso de ignición, el cual se define como el tiempo que transcurre entre el momento en que se inyecta el combustible y el momento en que empieza la combustión. Para determinar ese tiempo se utilizó la ecuación de Hardenberg y Hase [27], la cual se puede expresar como:

$$
\begin{array}{r}
t_{i g n}=\left[\frac{\left(0.36+0.22 C_{m}\right)}{0.006 n_{m}}\right] \cdot \exp \left[E A \left(\frac{1}{R_{u} T}\right.\right. \\
\left.\left.-\frac{1}{17190}\right)\left(\frac{21.2}{p-12.4}\right)^{0.63}\right]
\end{array}
$$

En esta ecuación $E A$ es la energía de activación aparente, la cual se define como:

$$
E A=\frac{618840}{C N+25}
$$

\subsubsection{Calor transferido a las paredes}

El cálculo del calor transferido a las paredes del cilindro se realizó por medio de la ecuación de Woschni [28], la cual abarca la convección y la radiación en un solo coeficiente:

$$
\begin{aligned}
& \frac{d Q_{w}}{d t}=h_{g} A\left(\mathrm{~T}-\mathrm{T}_{\mathrm{w}}\right) \\
& h_{g}=3,26 D^{-0,2} P^{0,8} T^{-0,55}\left[C_{1} C_{m}\right. \\
& \left.\quad+\frac{C_{2} V_{d} T_{r e f}}{P_{\text {ref }} V_{\text {ref }}}\left(P-P_{m}\right)\right]^{0,8}
\end{aligned}
$$

Los coeficientes de la ecuación de Woschni $\left(C_{1}\right.$ y $\left.C_{2}\right)$ fueron tomados de acuerdo con 1 proceso considerado, tal como se ve en la tabla 1 .

Tabla 1. Coeficientes de la ecuación de Woschni de acuerdo con el proceso considerado.

\begin{tabular}{|l|l|l|}
\hline Parte del ciclo & $\mathbf{C}_{\mathbf{1}}$ & $\mathbf{C}_{\mathbf{2}}$ \\
\hline Renovación de carga & 6,18 & 0 \\
\hline Compresión & 2,28 & 0 \\
\hline Combustión/Expansión & 2,28 & 0,00324 \\
\hline
\end{tabular}

Fuente: referencia [8].

Para determinar la temperatura media de las paredes se utilizó la ecuación planteada por Armas [29]: 


$$
T_{w}=T_{r f}+a \cdot\left(\rho_{c} \cdot c_{m}\right)^{0,8}+b \cdot p m e
$$

Donde a y b son las constantes de ajuste.

\subsubsection{Volumen del cilindro $y$ área de transferencia de calor}

A partir de la geometría del cilindro del motor fueron calculados el volumen instantáneo de la cámara de combustión $V(\theta)$, el área de transferencia de calor $A(\theta)$ y la variación del volumen de la cámara con respecto al ángulo de avance del cigüeñal $\frac{d V(\theta)}{d \theta}$. De este modo se tiene que [30]:

$$
\begin{aligned}
& V(\theta)=\frac{\pi}{180}\left(V_{d}\left[\frac{1-\cos \theta}{2}+\frac{L}{S}-\frac{1}{2} \sqrt{\left(\frac{2 L}{S}\right)^{2}-(\operatorname{sen} \theta)^{2}}\right]\right. \\
& \left.+\frac{V_{d}}{(r-1)}\right) \\
& A(\theta)=\frac{\pi D^{2}}{4}+\frac{4 V(\theta)}{D} \\
& \frac{d V(\theta)}{d \theta}=\frac{V_{d}}{S}\left[\frac{\operatorname{sen} 2 \theta}{2 \sqrt{\left(\frac{2 L}{S}\right)^{2}-(\operatorname{sen} \theta)^{2}}}+\operatorname{sen} \theta\right]
\end{aligned}
$$

\subsubsection{Relación de calores específicos de la mezcla de gases}

Ya que en este trabajo se consideró al cilindro como un sistema cerrado con adición de calor, para el cálculo de la relación de calores específicos se asumió que el gas en la cámara de combustión está compuesto por una mezcla de aire y gases residuales. Por lo tanto, esa relación, para cada ángulo de giro del cigüeñal, se calculó mediante la ecuación:

$$
\gamma(\theta)=\frac{[1-(m f b(\theta)+f)] C_{p a}+(m f b(\theta)+f) C_{p r}}{[1-(m f b(\theta)+f)] C_{v a}+(m f b(\theta)+f) C_{v r}}
$$

Donde los subíndices a y $\mathrm{r}$ hacen referencia al aire y a los gases residuales, respectivamente. Los calores específicos $\left(C_{p a}, C_{p r}, C_{v a}\right.$ y $\left.C_{v r}\right)$ se calcularon utilizando la ecuación (12) para cada una de las especies que componen al aire y a los gases residuales.

$$
\frac{c_{p i}(T)}{R}=a_{i 1}+a_{i 2} T+a_{i 3} T^{2}+a_{i 4} T^{3}+a_{i 5} T^{4}
$$

En esta ecuación el subíndice i denota la especie considerada. Los coeficientes $\boldsymbol{a}_{i 1}$ a $\boldsymbol{a}_{i 5}$ se encuentran en varias fuentes, en este caso fueron tomados de [31]. Al realizar este cálculo se asumió que los gases residuales producto de la combustión están compuestos por cuatro especies: $\mathrm{O}_{2}, \mathrm{~N}_{2}, \mathrm{CO}_{2}$ y $\mathrm{H}_{2} \mathrm{O}$; mientras que el aire se consideró como una mezcla de $\mathrm{O}_{2}$ y $\mathrm{N}_{2}$.

\subsubsection{Trabajo bruto indicado por ciclo}

Este es el trabajo que realiza el motor en las carreras de compresión y combustión/expansión. Para determinarlo, en cada cilindro se calculó el área bajo la gráfica presión contra volumen del cilindro, la cual viene dada por la ecuación (13).

$$
W_{c, i g}=\oint p d V
$$

Dicha ecuación fue resuelta mediante la regla del trapecio.

\subsubsection{Potencia indicada}

Para calcularla se utilizó la siguiente ecuación [8]:

$$
\boldsymbol{P}_{\boldsymbol{i}}=\frac{\boldsymbol{W}_{\boldsymbol{c}, \boldsymbol{i} \boldsymbol{g}} \boldsymbol{n}_{\boldsymbol{m}}}{\boldsymbol{n}_{\boldsymbol{R}}}
$$

Donde $\mathrm{n}_{\mathrm{R}}$ es el número de giros del cigüeñal en un ciclo, para un motor de cuatro tiempos $n_{R}=2$.

\subsubsection{Eficiencia térmica indicada}

Esta es la relación entre el trabajo bruto indicado y la energía necesitada para desarrollar este trabajo, por lo tanto, viene determinada por:

$$
\eta_{t e r}=\frac{W_{c, i g}}{m_{f} P C I}
$$

\subsection{Validación del modelo}

Como fue ya mencionado, el modelo desarrollado en este trabajo es adecuado para predecir el desempeño de motores diésel alimentados con cualquier tipo de combustible hidrocarburo, siempre y cuando se conozca su fórmula molecular $\left(\mathrm{C}_{\mathrm{m}} \mathrm{H}_{\mathrm{n}} \mathrm{O}_{\mathrm{p}}\right)$ y sus propiedades. Con el fin de comprobar la efectividad del modelo, este fue validado por comparación con datos experimentales de presión en cámara de dos motores, a saber, un motor monocilíndrico Agrale M95, alimentado con mezcla de diésel y biodiésel de aceite de palma B3 (3\% de biodiésel y $97 \%$ de diésel, en volumen) y biodiésel puro (B100), y un motor de cuatro cilindros Isuzu A-4JA1, alimentado con combustible $\mathrm{B} 10$ (10\% de biodiésel y $90 \%$ de diésel) y mezclas de combustible B10 con bio-oil hidrotratado, obtenido sintéticamente, siguiendo la metodología utilizada por Pinheiro [4], al $1 \%$ y $2 \%$ en volumen 
(B10BOH1 y B10BOH2); las características de los motores y combustibles básicos utilizados se presentan en las tablas 2 y 3 , respectivamente. En la tabla 3, las propiedades del diésel puro, bio-oil hidrotratado y biodiésel fueron obtenidas de los trabajos de Jagadish et al. [32], de Conti et al. [33] y Colaço et al. [15], respectivamente.

Tabla 2. Especificaciones de los motores utilizados.

\begin{tabular}{|l|c|c|}
\hline Modelo & Agrale-M95 & Isuzu A-4JA1 \\
\hline Tipo & $\begin{array}{c}\text { Enfriado por } \\
\text { agua, 4 } \\
\text { tiempos }\end{array}$ & $\begin{array}{c}\text { Enfriado por } \\
\text { agua, 4 } \\
\text { tiempos }\end{array}$ \\
\hline $\begin{array}{l}\text { Dimensiones }(\mathrm{L} \times \\
\mathrm{W} \times \mathrm{H})\end{array}$ & $\begin{array}{c}683 \times 575 \times \\
702(\mathrm{~mm})\end{array}$ & $\begin{array}{c}805 \times 625 \times \\
729(\mathrm{~mm})\end{array}$ \\
\hline $\begin{array}{l}\text { Número de } \\
\text { cilindros }\end{array}$ & 1 & 4 \\
\hline $\begin{array}{l}\text { Volumen } \\
\text { desplazado }\end{array}$ & $0,774 \mathrm{dm}^{3}$ & $2,449 \mathrm{dm}^{3}$ \\
\hline $\begin{array}{l}\text { Relación de } \\
\text { compresión }\end{array}$ & $21: 1$ & $18,4: 1$ \\
\hline $\begin{array}{l}\text { Diámetro x } \\
\text { carrera }\end{array}$ & $95 \times 105 \mathrm{~mm}$ & $93 \times 92 \mathrm{~mm}^{-1}$ \\
\hline $\begin{array}{l}\text { Sistema de } \\
\text { inyección }\end{array}$ & $\begin{array}{c}\text { Inyección } \\
\text { directa }\end{array}$ & $\begin{array}{c}\text { Inyección } \\
\text { directa }\end{array}$ \\
\hline $\begin{array}{l}\text { Avance en la } \\
\text { inyección }\end{array}$ & $17^{\circ}$ & $14^{\circ}$ \\
\hline
\end{tabular}

Fuente: autores.

Tabla 3. Propiedades de los combustibles.

\begin{tabular}{|l|l|l|l|}
\hline Propiedad & diésel & $\begin{array}{l}\text { bio-oil } \\
\text { hidrotratado }\end{array}$ & biodiésel \\
\hline $\begin{array}{l}\text { Molecular } \\
\text { formula }\end{array}$ & $\mathrm{C} 10 \mathrm{H} 2$ & $\begin{array}{l}\mathrm{C} 10 \mathrm{H} 13,34 \\
\mathrm{O} 2,53\end{array}$ & $\begin{array}{l}\mathrm{C} 19 \mathrm{H} 38,62 \\
\mathrm{O} 1,82\end{array}$ \\
\hline Densidad & $\begin{array}{l}840 \\
\mathrm{~kg} / \mathrm{m} 3\end{array}$ & $1070 \mathrm{~kg} / \mathrm{m} 3$ & $871,9 \mathrm{~kg} / \mathrm{m} 3$ \\
\hline $\begin{array}{l}\text { Poder } \\
\text { calorífico } \\
\text { inferior }\end{array}$ & $\begin{array}{l}42,5 \\
\mathrm{MJ} / \mathrm{kg}\end{array}$ & $28,5 \mathrm{MJ} / \mathrm{kg}$ & $36,78 \mathrm{MJ} / \mathrm{kg}$ \\
\hline $\begin{array}{l}\text { Número de } \\
\text { cetano }\end{array}$ & 45 & ------ & 59,3 \\
\hline
\end{tabular}

Fuente: referencias [32], [33] y [15].

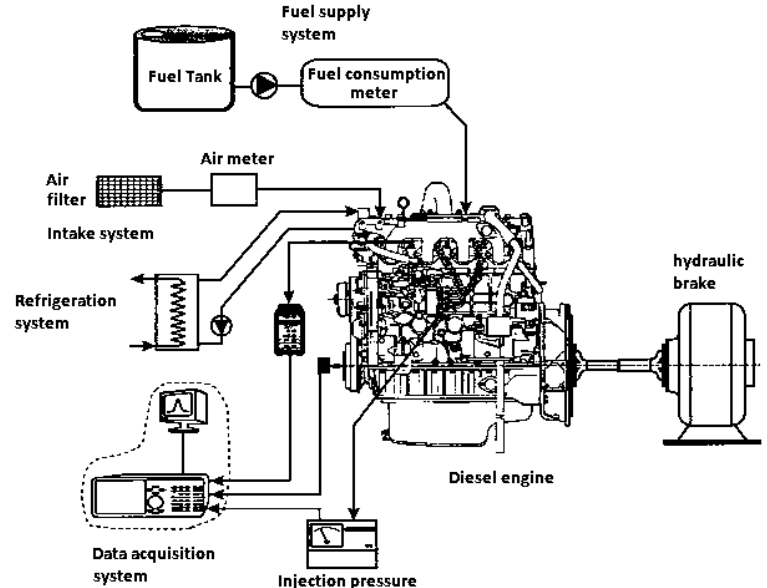

Figura. 1. Configuración experimental. Fuente: referencia [34].

Los datos experimentales de presión en cámara de motor Agrale M95 fueron tomados del trabajo de Colaço et al. [15], mientras que los del motor Isuzu A-4JA1 fueron tomados en el banco de ensayos de motores de la Universidad de Antioquia, en Medellín, Colombia (véase figura $1[34])$.

\section{Resultados}

\subsection{Validación con datos del motor Agrale M95}

La comparación entre los datos experimentales y simulados de este motor, tras ajustar los parámetros de Wiebe, se llevó a cabo a tres velocidades de operación, $1500 \mathrm{rpm}, 2000 \mathrm{rpm}$ y $2500 \mathrm{rpm}$, y dos condiciones de carga, $20 \mathrm{Nm}$ y $30 \mathrm{Nm}$, para un total de seis puntos de operación evaluados para cada combustible (B3 y B100). En la figura 2 se presenta esta comparación en uno de los puntos mencionados, $2000 \mathrm{rpm}$ y $30 \mathrm{Nm}$, para los dos combustibles evaluados; allí se puede ver, por medio de gráficas semilogarítmicas, la variación de la presión en función de la relación entre el volumen instantáneo y el volumen máximo del cilindro. Como se puede observar, hay una buena concordancia entre los datos modelados y los experimentales, condición que se repite en los demás puntos de operación. En estas pruebas el error relativo promedio de los valores de presión en el ciclo simulado fue de $5,86 \%$ y el error relativo promedio de los valores de presión pico simulada fue de $0,5 \%$, lo cual demuestra la eficacia del modelo desarrollado. 
B3 (2000 rpm - 30Nm)

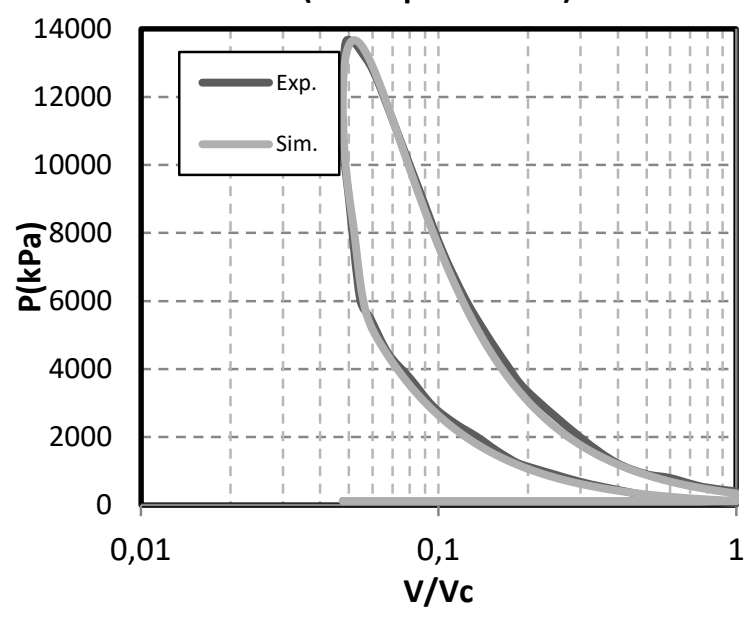

a)

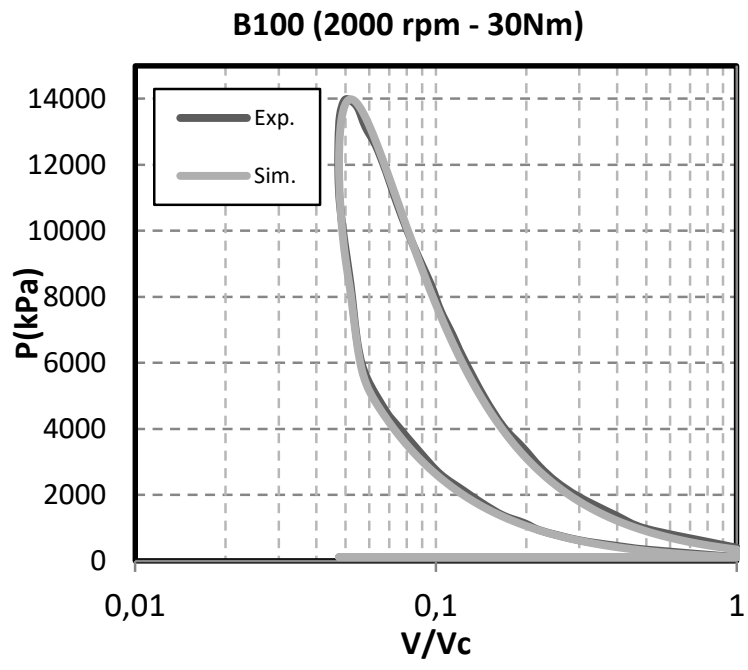

b)

Figura. 2. Comparación entre las curvas experimentales y simuladas del motor Agrale a $2000 \mathrm{rpm}$ y 30N.m. a) Motor alimentado con combustible B3, b) Motor alimentado con combustible B100. Fuente: autores.

\subsection{Validación con datos del motor Isuzu A-4JA1}

Al realizar las pruebas con este motor, la presión en el cilindro se tomó con el motor operando a 2000 rpm y 25 $\mathrm{Nm}$, y $2000 \mathrm{rpm}$ y $50 \mathrm{Nm}$. En la figura 3 se presenta la comparación entre las presiones simuladas y experimentales obtenidas en el motor operando a 2000 rpm y $25 \mathrm{Nm}$, y alimentado con las tres mezclas de combustibles (B10, B10BOH1 y B10BOH2), esto por medio de gráficas semilogarítmicas de presión contra la relación entre el volumen instantáneo y el volumen máximo del cilindro. $\mathrm{Al}$ igual que con las pruebas hechas en el motor Agrale, en este caso se observó una buena concordancia entre los datos modelados y los experimentales de las diferentes pruebas, con lo cual se obtuvo un error relativo promedio del $7 \%$ para los datos de presión del ciclo simulado, y del 1\%, para los datos de presión pico. En la figura 3 también se observa que el biooil hidrotratado mezclado en porciones pequeñas, del 1 $\%$ y $2 \%$, no genera cambios apreciables en la presión, las diferencias allí mostradas se deben más a las condiciones del experimento que al porcentaje de mezcla, esto se observa también en las otras pruebas hechas en este motor.

\subsection{Efecto del bio-oil hidrotratado en el desempeño del motor}

Para conocer los efectos del bio-oil hidrotratado en el desempeño del motor se utilizó el modelo desarrollado para simular y comparar los valores de presión en cámara, potencia indicada y eficiencia térmica indicada que se obtendrían en un motor Agrale M95, alimentado con combustible diésel convencional, bio-oil hidrotratado (BOH) y dos mezclas diésel/bio-oil hidrotratado en proporciones de $80 \%$ de diésel y $20 \%$ de bio-oil hidrotratado (BOH20), y 50\% de diésel y $50 \%$ de bio-oil hidrotratado (BOH50), en volumen. Las características del motor utilizado y las propiedades del diésel convencional y del bio-oil hidrotratado se presentan en la tabla 2 y 3 , respectivamente. El número de cetano del bio-oil hidrotratado se asumió igual al número de cetano del diésel puro [45]; esta aproximación puede generar ciertas variaciones al calcular el tiempo de retraso en la ignición, pero lleva a un error pequeño a la hora de calcular la variación de la presión en la cámara de combustión.

A continuación se presenta la comparación entre los valores de presión, la potencia indicada y la eficiencia térmica indicada obtenidos para el dieésel, el bio-oil hidrotratado y las respectivas mezclas entre ellos (BOH20 y BOH50). 


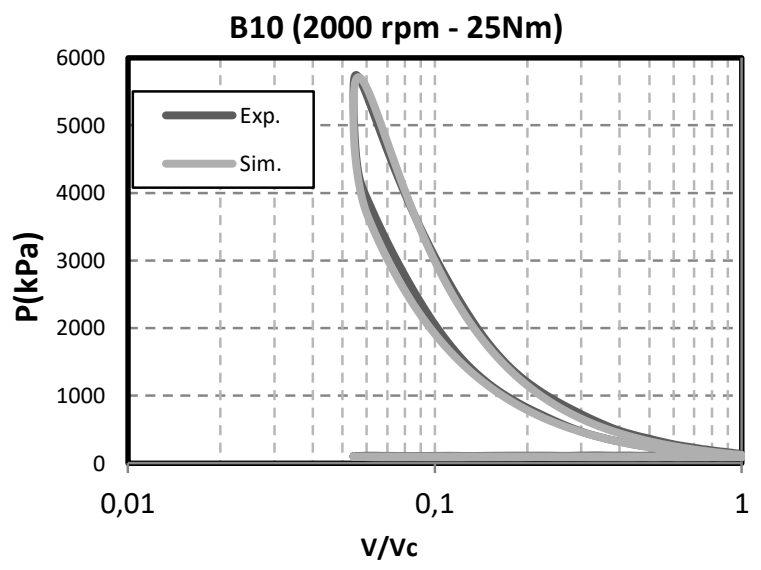

a)

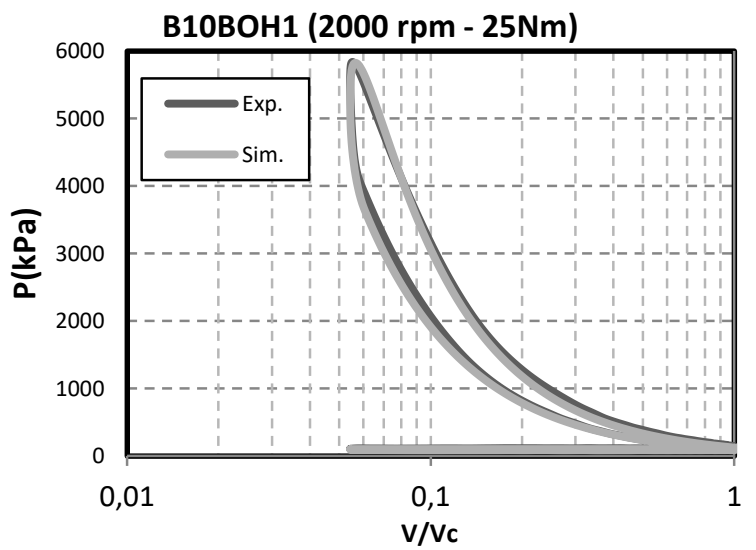

b)

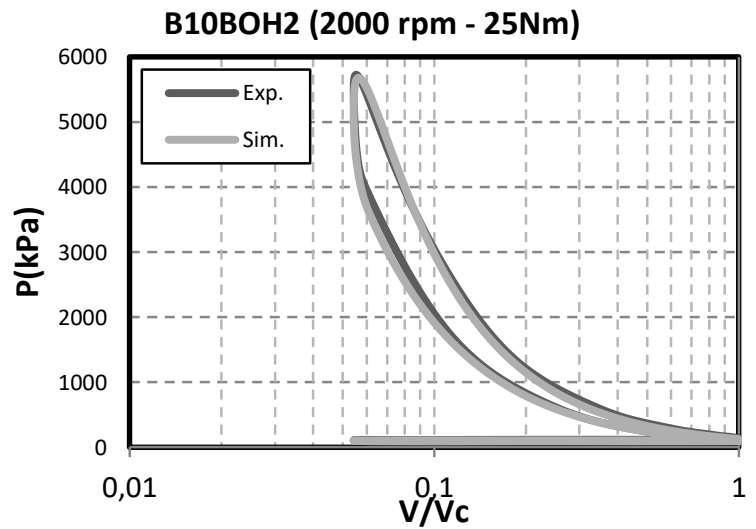

c)

Figura 3. Comparación entre las curvas experimentales y simuladas del motor Isuzu a $2000 \mathrm{rpm}$ y 25 N.m. a) Motor alimentado con combustible B10, b) Motor alimentado $99 \%$ de combustible B10 y $1 \%$ de bio-oil hidrotratado, c) Motor alimentado $98 \%$ de combustible B10 y $2 \%$ de bio-oil hidrotratado. Fuente: autores. 


\subsubsection{Diagrama P- $\theta$}

En la figura 4 se presenta la comparación de la evolución de presión de los diferentes combustibles considerados con el motor operando a alta carga y un régimen de 2000 rpm, en los demás puntos de operación del motor se observa la misma tendencia allí presentada. El diagrama presión contra ángulo de giro del cigüeñal puede ser usado para conocer el comportamiento termodinámico del motor, ya que permite conocer la evolución de presión en la cámara de combustión al utilizar un determinado combustible. En el análisis realizado se encontró que el pico de presión es más alto cuando se utiliza diésel convencional en el motor que cuando se utiliza bio-oil hidrotratado y sus mezclas con diésel; esto se atribuye al menor poder calorífico del bio-oil hidrotratado. Como se observa en la figura, la diferencia entre los picos de presión depende directamente de la cantidad del bio-oil hidrotratado mezclado con el diésel, y se alcanzan los valores límites de presión al utilizar $\mathrm{BOH}$ y diésel puros.

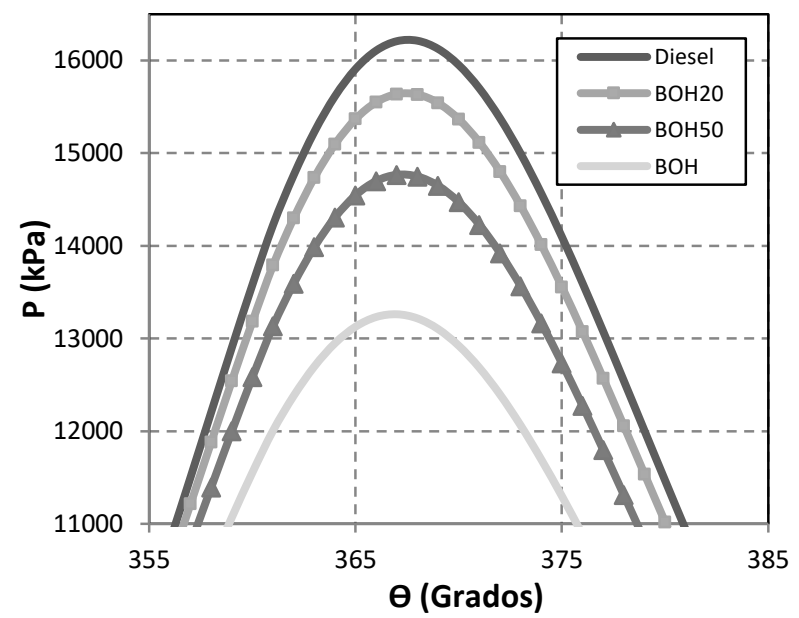

Figura. 4. Presión versus ángulo de giro del cigüeñal para mezclas diésel/bio-oil hidrotratado con el motor operando a alta carga y 2000 rpm. Fuente: autores.

\subsubsection{Potencia indicada}

Al igual que con la presión, se evidenció una disminución en la potencia indicada al utilizar bio-oil hidrotratado y sus mezclas, esto debido al menor trabajo bruto indicado desarrollado en cada caso. Sin embargo, se presentan valores cercanos a los del diésel cuando se utiliza la mezcla $\mathrm{BOH} 20$, la misma tendencia se observa para todas las cargas y velocidades del motor. En la figura 5 se muestra la potencia indicada de los diferentes combustibles para el motor operando a diferentes velocidades y un dosado (F/A) igual a 0,035.

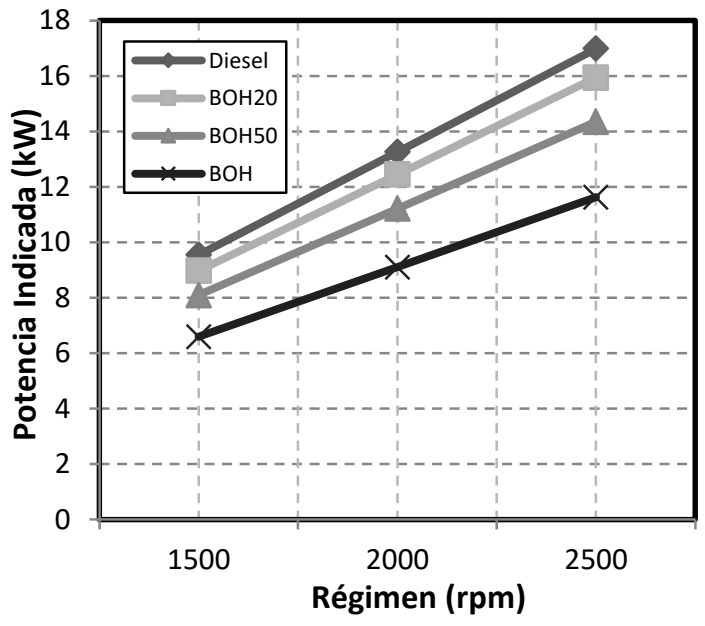

Figura 5. Potencia indicada del motor a diferentes velocidades. Fuente: autores.

\subsubsection{Eficiencia térmica indicada}

La eficiencia térmica indicada es la relación entre el trabajo bruto indicado y la entrada de energía brindada por el combustible, en el caso estudiado se encontró que en la mayor parte del rango de operación la eficiencia térmica del bio-oil hidrotratado es mayor que la del diésel convencional; esto se debe a la presencia de oxígeno en el bio-oil hidrotratado, el cual mejora la combustión, y al menor poder calorífico de este, lo cual hace que la relación entre el trabajo desarrollado y la entrada de energía aumente pese al mayor trabajo desarrollado por el diésel. El efecto de las propiedades de bio-oil hidrotratado sobre el aumento en la eficiencia térmica indicada disminuyen al disminuir la carga del motor, hasta el punto que para condiciones de baja carga la eficiencia térmica indicada llega a ser mayor para el diésel que para el bio-oil hidrotratado. En la figura 6 se pude apreciar la eficiencia térmica indicada del motor a $1500 \mathrm{rpm}$ y tres tipos de dosado: 0,015, 0,035 y 0,055.

La figura 7 muestra la eficiencia térmica indicada del motor operando con un dosado de 0,055 y diferentes velocidades. Como era de esperarse, se puede observar que al aumentar la velocidad crece la eficiencia térmica indicada, manteniéndose siempre más baja la eficiencia térmica del diésel que la de los demás combustibles. 


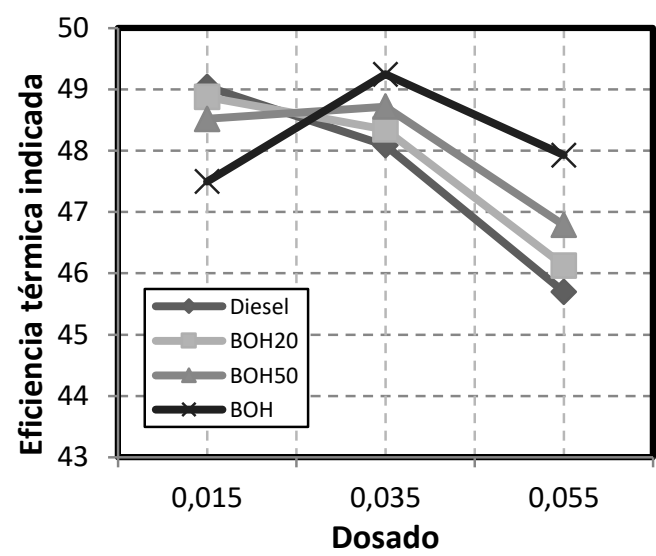

Figura 6. Eficiencia térmica indicada del motor con diferentes dosados. Fuente:autores.

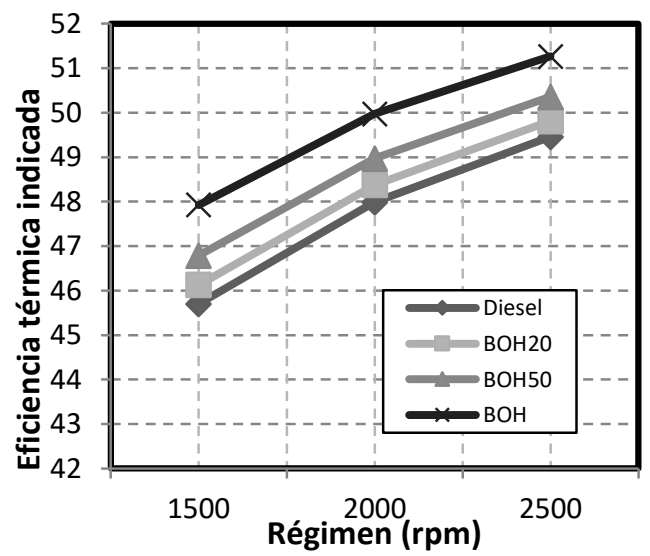

Figura 7. Eficiencia térmica indicada del motor a diferentes velocidades. Fuente: autores.

\section{Conclusiones}

El bio-oil hidrotratado puede convertirse en un sustituto parcial del combustible diésel derivado del petróleo, ya que, al mezclarse con este último en pequeñas proporciones cercanas al $20 \%$, se presenta un desempeño similar en el motor, pero sin contribuir a los problemas ambientales y sociales asociados con los combustibles derivados del petróleo o con los biocombustibles de primera generación.

El poder calorífico inferior del bio-oil hidrotratado es el principal factor que contribuye al menor desarrollo de potencia en el motor, en comparación con el diésel convencional. Por lo tanto, al mejorar este factor se podrían lograr mejores resultados al utilizarlo como combustible sustituto para motores diésel.

Se deben realizar estudios experimentales más completos con concentraciones de bio-oil hidrotratado mayores al 1 y al $2 \%$, en donde se estudien otros efectos de este combustible sobre el motor, como las emisiones de contaminantes, para así poder concluir de manera definitiva el efecto que este tendrá sobre la operación del motor.

\section{Agradecimientos}

Los autores expresan sus agradecimientos a las Vicerrectoría de Investigación y Extensión de la Universidad Industrial de Santander por la financiación del proyecto Producción de energía a partir de residuos provenientes de la transformación de la biomasa, del cual hace parte este trabajo.

\section{Referencias}

[1] F. Serna, L. Barrera, and H. Montiel, "Impacto Social y Económico en el uso de Biocombustibles," $J$ Technol Manag Innov, vol. 6, no. 1, pp. 100-114, 2011.

[2] M. A. Altieri, "Los impactos ecológicos de los sistemas de producción de biocombustibles a base de monocultivos a gran escala en América," Agroecología, vol. 4, pp. 59-67, 2009.

[3] N. Hackenberg, "Biocombustibles de Segunda Generación," Rev Virtual REDESMA, vol. 2, no. 2, pp. 49-61, Jul. 2008.

[4] A. Pinheiro, "Influence des composés oxygénés issus de la biomasse lignocellulosique et de leurs produits d'hydrodéoxygénation sur les cinétiques des réactions d'hydrotraitement de gazoles," Tesis Doctoral, Université Claude Bernard, Lyon, France, 2008.

[5] A. S. Ramadhas, S. Jayaraj, and C. Muraleedharan, "Theoretical modeling and experimental studies on biodiesel-fueled engine," Renewable Energy, vol. 31, no. 11, pp. 1813-1826, Sep. 2006.

[6] T. K. Gogoi and D. C. Baruah, "A cycle simulation model for predicting the performance of a diesel engine fuelled by diesel and biodiesel blends," Energy, vol. 35, no. 3, pp. 1317-1323, Mar. 2010.

[7] T. Ganapathy, P. Gakkhar, and K. Murugesan, "An analytical and experimental study of performance on jatropha biodiesel engine," Thermal Science, vol. 13, no. 3, pp. 69-82, 2009.

[8] J. Heywood, Internal Combustion Engine Fundamentals, 1 st ed. McGraw-Hill Science/Engineering/Math, 1988. 
[9] G. F. García Sánchez, J. L. Chacón Velasco, and A. Chaves Guerrero, "Modelado de la combustión en motores Diésel: revisión del estado del arte," REVISTA ION, vol. 26, no. 1, Nov. 2013.

[10]E. Gutiérrez, "Modelado numérico de la atomización y evaporación de un chorro diesel mediante análisis CFD," Tesis de Maestría, Universidad de Antioquia, Medellín, 2008.

[11]A. L. Delgado-Mejia, L. C. Olmos-Villalba, and S. E. Rivero-Mejia, "Comparative study of the indicated cycle of a diesel engine using simulation CFD and experimental data," Rev. UIS Ing., vol. 13, no. 1, pp. 2331,2014 .

[12]J. Agudelo, A. Agudelo, and P. Benjumea, "Estudio de chorros diesel usando mecánica de fluidos computacional," Rev fac ing univ Antioquia, no. 49, pp. 61-69, Sep. 2009.

[13]A. Delgado, "Simulación numérica de los procesos al interior del cilindro en un motor diesel mediante análisis CFD," Tesis de Maestría, Universidad de Antioquia, Medellín, 2010.

[14]A. V. Bueno, J. A. Velásquez, and L. F. Milanez, "Heat release and engine performance effects of soybean oil ethyl ester blending into diesel fuel," Energy, vol. 36, no. 6, pp. 3907-3916, Jun. 2011.

[15]M. J. Colaço, C. V. Teixeira, and L. M. Dutra, "Thermal analysis of a diesel engine operating with diesel-biodiesel blends," Fuel, vol. 89, no. 12, pp. 37423752, Dec. 2010.

[16]Sanjay Patil and M.M.Akarte, "Investigation on Effect of Variation in Compression Ratio on Performance and Combustion Characteristics of C.I Engine Fuelled With Palm Oil Methyl Ester (POME) and its Blends by Simulation," Global Journal of Researches in Engineering - Automotive Engineering, vol. 12, no. 2, pp. 34-41, 2012.

[17]G. Kökkülünk, G. Gonca, and A. Parlak, "The Effects of Design Parameters on Performance and NO Emissions of Steam-Injected Diesel Engine with Exhaust Gas Recirculation," Arab J Sci Eng, vol. 39, no. 5, pp. 4119-4129, Mar. 2014.

[18]S. P. Potdukhe and M. M. Deshmukh, "Modeling and Energy Analysis of a Diesel and Biodiesel Fuelled Engine," International Journal of Science and Research (IJSR), vol. 4, no. 5, May 2015.
[19]T. Naitam and M. M. Deshmukh, "Combustion Modeling of Biodiesel Fuelled Direct Injection CI Engine," International Journal of Science and Research (IJSR), vol. 4, no. 5, pp. 288-293, May 2015.

[20]M. Mikulski and S. Wierzbicki, "Numerical investigation of the impact of gas composition on the combustion process in a dual-fuel compression-ignition engine," Journal of Natural Gas Science and Engineering, vol. 31, pp. 525-537, Apr. 2016.

[21]F. A. Williams, Combustion Theory: Second Edition, Second Edition. Westview Press, 1994.

[22]G. M. Faeth, "Spray combustion phenomena," Symp (Int) Combust, vol. 26, no. 1, pp. 1593-1612, 1996.

[23]F. Payri and J. M. Desantes, Motores de combustión interna alternativos, 1st ed. España: Reverte, 2011.

[24]M. Lapuerta, R. Ballesteros, and J. R. Agudelo, "Effect of the gas state equation on the thermodynamic diagnostic of diesel combustion," Appl Therm Eng, vol. 26, no. 14-15, pp. 1492-1499, Oct. 2006.

[25]F. Payri, P. Olmeda, J. Martín, and A. García, “A complete 0D thermodynamic predictive model for direct injection diesel engines," Applied Energy, vol. 88, no. 12, pp. 4632-4641, Dec. 2011.

[26]N. Miyamoto, T. Chikahisa, T. Murayama, and R. Sawyer, "Description and Analysis of Diesel Engine Rate of Combustion and Performance Using Wiebe's Functions," SAE International, Warrendale, PA, 850107 , Feb. 1985

[27]H. O. Hardenberg and F. W. Hase, "An Empirical Formula for Computing the Pressure Rise Delay of a Fuel from Its Cetane Number and from the Relevant Parameters of Direct-Injection Diesel Engines," SAE International, Warrendale, PA, 790493, Feb. 1979.

[28]G. Woschni, “A Universally Applicable Equation for the Instantaneous Heat Transfer Coefficient in the Internal Combustion Engine," SAE International, Warrendale, PA, 670931, Feb. 1967.

[29]O. Armas Vergel and F. P. González, Diagnóstico experimental del proceso de combustión en motores diesel de inyección directa. Universidad Politécnica de Valencia, 1998. 
[30]C. R. Ferguson and A. T. Kirkpatrick, Internal Combustion Engines: Applied Thermosciences, 2nd ed. Wiley, 2000.

[31]R. S. Benson, Advanced Engineering Thermodynamics, 2nd Revised edition. Pergamon Press, 1977.

[32]D. Jagadish, R. Kurmar, and M. K., "Zero dimensional simulation of Combustion Process of a DI Diesel engine fuelled with Biofuels," WASET, vol. 80, pp. 819-825, 2011.

[33]L. Conti, G. Scano, J. Boufala, and S. Mascia, "Biocrude oil hydrotreating in a continuous bench-scale plant," Dev Thermochem Biomass Convers, vol. 1, pp. $622-632$.

[34]A. F. Agudelo, J. R. Agudelo, and P. N. Benjumea, Diagnostico de la combustion de biocombustibles en motores. Universidad de Antioquia, 2007. 